\title{
DISCRETE CRACK GROWTH ANALYSIS METHODOLOGY
FOR THROUGH CRACKS IN PRESSURIZED FUSELAGE STRUCTURES
}

\author{
David O. Potyondy \\ Georgia Institute of Technology \\ Computational Modeling Center \\ Atlanta, GA
Paul A. Wawrzynek and Anthony R. Ingraffea Cornell University Fracture Group Ithaca, NY

N95- 19473

113057

\section{SUMMARY}

\begin{abstract}
A methodology for simulating the growth of long through cracks in the skin of pressurized aircraft fuselage structures is described. Crack trajectories are allowed to be arbitrary and are computed as part of the simulation. The interaction between the mechanical loads acting on the superstructure and the local structural response near the crack tips is accounted for by employing a hierarchical modeling strategy. The structural response for each cracked configuration is obtained using a geometrically nonlinear shell finite element analysis procedure. Four stress intensity factors, two for membrane behavior and two for bending using Kirchhoff plate theory, are computed using an extension of the modified crack closure integral method. Crack trajectories are determined by applying the maximum tangential stress criterion. Crack growth results in localized mesh deletion, and the deletion regions are remeshed automatically using a newly developed all-quadrilateral meshing algorithm. The effectiveness of the methodology and its applicability to performing practical analyses of realistic structures is demonstrated by simulating curvilinear crack growth in a fuselage panel that is representative of a typical narrow-body aircraft. The predicted crack trajectory and fatigue life compare well with measurements of these same quantities from a full-scale pressurized panel test.
\end{abstract}

\section{INTRODUCTION}

Concern for the safety of aging airliners motivates the development of a methodology for simulating the behavior of long through cracks in the skin of pressurized fuselages wherein crack trajectory is not known a priori. Long cracks in the fuselage skin can arise from accidental damage during flight, e.g., the failure of an engine component resulting in a puncture of the skin, or from fatigue crack growth whereby several smaller cracks along a row of rivets might have linked up. The cabin of a passenger aircraft is pressurized. Since the loading from pressurization is cyclic, with one pressure cycle for each flight, older aircraft can accumulate significant numbers of cycles and substantial fatigue crack growth. Regardless of the cause of crack formation, simulation, along with experimental testing, can verified simulation metter understanding of the behavior of such cracks. Also, an experimentally aircraft damage tolerance. 
Crack growth simulations, in general, are difficult to perform using existing codes for computational solid mechanics alone. The difficulty lies in the need for a continual updating of the geometry, as well as the discretization of the structure, as a result of crack initiation and propagation. Most available codes will not allow the geometry of the structure to evolve automatically during the course of a simulation, thereby forcing the analyst to perform tedious and time-consuming manual updates to the model for each step of crack growth. These difficulties can be overcome by use of a single fracture simulation program containing a representational model of the problem that separates the problem description from the problem discretization. Such a program provides the necessary framework for the entire simulation process, employing an existing computational solid mechanics module simply as an analysis engine whenever the structural response of a particular cracked configuration is necessary. The representational model consists of a geometrical model to which simulation attributes, such as loads and boundary conditions, can be attached once at the start of the simulation. In this way, information is inherited automatically by each computational mesh that is generated during crack growth. The primary components of this discrete crack growth analysis methodology are shown in Figure 1. It has been implemented within the FRANC3D fracture simulation program [Wawrzynek, et al. (1988); Martha (1989); Wawrzynek (1991)], a general software framework that supports simulation of curvilinear discrete crack propagation in stiffened, thin shell structures [Potyondy (1993)] and non-planar discrete crack propagation in solid structures [Potyondy, et al. (1992); Martha, et al. (1993); Gray, et al. (1994)].

The discrete crack growth analysis methodology described here utilizes the FRANC3D software framework. It accounts for the dominant influence factors affecting crack growth behavior and integrates these with a set of fracture parameters that characterize the fracture process. The mechanics considerations underlying the methodology are summarized in the next section. This is followed by a description of the modeling approach that supports simulating crack growth in realistic fuselage panels. Then, two example simulations are presented in which computed stress intensity factor histories are used to predict crack trajectory and fatigue life and a comparison is made with measurements from a fullscale pressurized panel test. A more thorough description of this work can be found in Potyondy (1993) and Potyondy, et al. (1994b).

\section{MECHANICS CONSIDERATIONS}

The behavior of long through cracks in the skin of pressurized fuselages is dominated by the following four factors [Rankin, et al. (1993)]: 1) a geometrically nonlinear stiffening effect (hereafter referred to as the bulge-out effect), caused by tensile membrane stresses in the direction parallel to the crack that restricts the crack edge bulging; 2) the mechanical loads, in addition to the internal pressure, acting on the superstructure that alter the stress distribution in the skin; 3) the presence of stiffening elements (frames and stringers) that alter locally the stress distribution in the skin; and, 4) plasticity effects around the crack tip. The first three factors can be modeled using a geometrically nonlinear shell finite element analysis procedure in which cracks are represented discretely in the mesh. In the present work, the nonlinear shell finite element code STAGS [Almroth, et al. (1986)], is used along with a hierarchical modeling strategy to account for the second factor. The effect of plasticity at the crack tips is ignored. (This effect is accounted for in the work of Shenoy, et al. (1994), who compute $T^{*}$, a relevant nonlinear fracture parameter, for typical fuselage structures. Refer to that paper for further details.)

The use of a geometrically nonlinear finite element shell model captures the global structural response by idealizing the fuselage as a cracked, stiffened, thin shell structure; however, additional modeling idealizations are required to predict crack trajectory and fatigue life. For cases in which the linear thin shell equations are applicable, stress intensity factors can be defined [Potyondy, et al. 
(1994b)]. However, in the present case, wherein geometrically nonlinear effects play a significant role in the shell behavior, there is no clear consensus about the proper definition of stress intensity factors.

Many workers in the field have adopted an engineering approach that restricts the generality of the solution procedure. First, only straight cracks, oriented and loaded symmetrically with respect to the stiffeners, are considered; thus, a single similitude parameter, the mode-I stress intensity factor $K_{I}$, can be used to correlate crack growth rate with experimentally obtained fatigue crack growth data. Also, crack trajectory is not considered, since the crack growth is assumed to be self-similar. The bulge-out effect is modeled by using a geometrically nonlinear solution procedure, and the energy release rate $G$, is computed. $K_{I}$ is obtained from the linear elastic, plane stress relation $K_{I}=\sqrt{E G}$ where $E$ is Young's

In contrast, the present methodology addresses the more general problem in which the cracks may be curved and need not be oriented or loaded symmetrically. Such cracks experience a combination of in-plane membrane loading and out-of-plane bending loading. The computed deformation pattern for such a case is shown in Figure 8. The unsymmetric out-of-plane bending could be called a nominal mode-III deformation. Hui and Zehnder (1993) propose a set of four stress intensity factors, $\left(k_{1}, k_{2}, K_{I}, K_{I I}\right)$, to account for this general loading. The mechanical behavior of the region near the crack tips is idealized using Kirchhoff plate theory to account for the bending loads and two-dimensional plane stress elasticity to account for the membrane loads. The stress intensity factors based on Kirchhoff plate theory are denoted by $k_{1}$ and $k_{2}$ while those based on two-dimensional elasticity theory are denoted by $K_{I}$ and $K_{I I}$. All four stress intensity factors have the units of force/length ${ }^{3 / 2}$. The four modes of loading are depicted in Figure 2. Note that the unsymmetric out-of-plane bending in Figure 8 is characterized by $k_{2}$.

The stress intensity factors described above arise from the assumptions of small deflection plate theory; however, a geometrically nonlinear shell finite element procedure is required to account for the bulge-out effect experienced by a long crack in a pressurized fuselage structure. In the present work, the engineering assumption is made that these stress intensity factors can also be used to characterize the fracture process for such structures. Thus, the stress intensity factors correspond to the equilibrium state obtained from the geometrically nonlinear shell computation. They are computed using an extension of the modified crack closure integral method [Potyondy (1993); Potyondy, et al. (1993)] that is implemented as a post-processing step to the shell finite element analysis. The individual components of the energy release rate $G_{i}$, are obtained from the nodal forces and displacements in the shell finite element mesh near the crack tip. These components are, in turn, related to the stress intensity factors via
the expressions:

$$
\begin{gathered}
G=\sum_{j=1}^{5} G_{j} \\
G_{1}=\frac{K_{I}^{2}}{E} \\
G_{2}=\frac{K_{I I}^{2}}{E} \\
G_{4}=\frac{k_{1}^{2} \pi}{3 E}\left(\frac{1+v}{3+v}\right)
\end{gathered}
$$




$$
G_{3}+G_{5}=\frac{k_{2}^{2} \pi}{3 E}\left(\frac{1+v}{3+v}\right)
$$

The stress intensity factors so obtained correspond to an equivalent model of a cracked Kirchhoff plate with the same energy release rate as that computed for the geometrically nonlinear problem.

No generally accepted criterion exists for predicting trajectories of cracks in pressurized thin shells. Existing criteria strictly apply for pure membrane stress conditions only. The stress state about a crack in a pressurized thin shell will always contain out-of-plane bending components, and additionally, these bending components will become large as the crack approaches stiffening elements. It is not yet apparent in what way these bending components are to be incorporated into an appropriate criterion. Rankin, et al. (1993) comment on this problem and refer to the summary of Zaal (1992); however, no suggestions are made for incorporating the bending components.

In this work, the crack front fields are described by $\left(k_{1}, k_{2}, K_{I}, K_{I I}\right)$. These fields apply to a crack in a plate wherein the crack front is assumed to be straight and aligned with the plate normal direction. (If the crack front were to deviate from this shape and orientation, a three-dimensional model of the crack front stress field involving $\left(K_{I}(s), K_{I I}(s), K_{I I I}(s)\right)$ would be required where $s$ parameterizes the crack front through the thickness.) Therefore, crack trajectory can be described fully by the path of a single point on the crack front at the shell midsurface. The path is described in terms of the instantaneous angle of crack propagation $\phi$, with which the crack will deviate from its current selfsimilar direction. $\phi$ will lie in the tangent plane to the midsurface at the crack tip. The maximum tangential stress theory [Erdogan and Sih (1963)] is used to predict $\phi$ via the relation

$$
K_{I} \sin \phi+K_{I I}(3 \cos \phi-1)=0
$$

Since $\phi$ is determined only at the shell midsurface, the two bending stress intensity factors do not enter into the relation.

\title{
MODELING APPROACH
}

\author{
Hierarchical Modeling Strategy
}

A hierarchical modeling strategy [Starnes and Britt (1991)] consisting of a series of models at differing scales provides a general mechanism for modeling pressurized fuselage structures by representing the important global and local features of a shell structure subjected to combined internal pressure and mechanical loads. The hierarchical modeling levels range from a relatively coarse global shell model for determining the internal load distribution and global response of the shell, to a highly refined local shell model for determining crack growth behavior.

The three hierarchical modeling levels employed in both of the simulations are depicted in Figure 3 and include a global shell model, a $6 \times 6$ bay stiffened panel model, and a $2 \times 2$ bay stiffened panel model. The global shell model can be subjected to internal pressure and mechanical loads that represent 
a bending moment, a vertical shear load, and a torsional load applied to the shell. A geometrically nonlinear analysis of the global shell model provides the internal load distribution for the shell as well as the kinematic boundary conditions (displacements and rotations) along the boundaries of the $6 \times 6$ bay stiffened panel model. In addition to these kinematic constraints, the $6 \times 6$ bay stiffened panel model is model provides a mernal pressure. A geometrically nonlinear analysis of the $6 \times 6$ bay stiffened panel well as the kinematic accurate determination of the interaction between skin, frames, and stiffeners as addition to these kinemation conditions along the boundaries of the $2 \times 2$ bay stiffened panel model. In pressure. A geometrically constraints, the $2 \times 2$ bay stiffened panel model is also subjected to internal (he $2 \times 2$ bay stiffened panel model provides the detailed force and deformation field near the crack tips used to compute $\left(k_{1}, k_{2}, K_{I}, K_{I I}\right)$ for successive crack lengths. An initial crack is present in all three of the hierarchical models, but the crack is grown only in the $2 \times 2$ bay stiffened panel model; thus, its kinematic boundary conditions are not recomputed during crack growth. All of the analyses are conducted using the STAGS shell finite element analysis code.

\section{Modeling Crack Growth}

All crack growth takes place in the $2 \times 2$ bay stiffened panel model that is constructed within FRANC3D and shown in Figure 4. The general steps of the process are illustrated in Figure 1. (In this case, the simulation attributes include the generalized displacements obtained from the 6x6 bay stiffened panel model and are attached to the corresponding edges along the boundary of the FRANC3D geometrical model.) During all stages of the simulation, the analyst can manipulate a perspective view of this model and its discretization interactively.

Once all of the simulation attributes have been attached to the geometrical model, the simulation process consists of iterating through the following steps: 1) A STAGS finite element model is generated from FRANC3D for the current cracked configuration. 2) After obtaining the equilibrium state by executing the STAGS code, the values of $\left(k_{1}, k_{2}, K_{I}, K_{I I}\right)$ are computed and are used to estimate crack growth direction. 3) The analyst determines the amount of crack extension at each crack tip. 4) The crack growth alters the topology of the FRANC3D geometrical model and leads to localized mesh deletion. 5) The deletion region is remeshed automatically using a newly developed all-quadrilateral element meshing algorithm [Potyondy, et al. (1994a)] which generates a graded mesh that is fine near the crack tips and coarser away from the crack tips. A four-noded, six degree-of-freedom per node, shell element [Rankin and Brogan (1991)] is used within the entire deletion region, including the area immediately adjacent to the crack tips. (The errors introduced in the stress intensity factor computation from element distortion are examined in Potyondy (1993). For the meshes used in the example simulations, the errors in $K_{I}$ and $K_{I I}$ are believed to be below 2 percent while the errors in $k_{1}$ and $k_{2}$ may reach 15 percent.) The process repeats with step 1 . Proceeding in this fashion, both the trajectory and the stress intensity factor history are determined.

\section{Generic Narrow-Body Panel}

A fuselage panel that is representative of a typical narrow-body aircraft has been modeled. The dimensions and description are from Miller, et al. (1992). The panel consists of 2024-T3 clad skin of thickness 0.036 inches, reinforced by stringers at 9.25 inch spacing, and frames, without shear-ties, at 20 inch spacing. Tear straps of thickness 0.036 inches run circumferentially at 10 inch spacing, centered on 
the frame and midway between frames, and longitudinally under the stringers.

The panel is comprised of the following structural components: skin, frames, stringers, tear straps, and stringer clips. These are shown in Figure 4. Typically, the tear straps are bonded directly to the skin whereas the other components are connected with rivets. In the work of Miller, et al. (1992) fastener flexibility was modeled using rigid body elements for rotational and axial displacements, and spring elements for transverse displacements. In the present work, fastener flexibility has not been modeled; it is assumed that there is a perfect bond between all structural components along their entire area of overlap.

Different modeling idealizations have been employed in the three models of the analysis hierarchy. In the global shell model, the skin is modeled with shell elements; the frames are modeled with shell elements to which beam elements are attached along some of the shell element edges; and, the stringers, tear straps, and stringer clips are modeled with beam elements. In the $6 \times 6$ bay stiffened panel model, all structural components are modeled with shell elements and beam elements are also included along the tops of the stringers. In the $2 \times 2$ bay stiffened panel model, these beam elements are replaced by shell elements that approximate the hooked shape. In both the $6 \times 6$ and $2 \times 2$ bay stiffened panel models, each layer in the overlap areas is modeled with its own separate shell elements by providing the eccentricity from a reference wall surface. The material properties used in the three models of the analysis hierarchy were a Poisson's ratio of 0.33 for all structural components; and, a Young's modulus of $10,500 \mathrm{ksi}$ for the skin and tear straps, and 10,700 ksi for the frames, stringers, and stringer clips.

\title{
EXAMPLE SIMULATIONS
}

\author{
Simulation 1: Trial Problem
}

The initial crack is located in a panel near the crown of the fuselage. It is a straight 6.0 inch crack oriented parallel to the stringer and centered on the midbay tear strap 2.31 inches from the stringer centerline. The crack cuts completely through both the tear strap and the skin. The boundary conditions for the global shell model are a uniform internal pressure of $8.0 \mathrm{psi}$ and a mechanical load consisting of a bending moment applied to the fuselage barrel. A plane of symmetry is assumed at one end of the barrel. The other end is restrained to remain in a plane and two equal but opposite point forces are applied at the top and bottom of the barrel. The point forces produce a moment of $5.65 \times 10^{6}$ inchpounds, directed so as to compress the fuselage crown. The applied moment is slightly less than the value that just causes buckling of the skin between frames in the fuselage crown. The crack is grown in one inch increments at each crack tip up to a total piecewise crack length of 16 inches. Both the global shell and the 6x6 bay stiffened panel models contain approximately 100,000 degrees of freedom. The FRANC3D models contain from 11,000 to 14,000 degrees of freedom.

The deformed structure for various crack lengths is shown in Figure 5. The computed crack trajectory, shown in Figure 6, appears reasonable. The crack is curving away from the nearest stringer. This is the observed behavior in pressurized panel tests of narrow-body fuselage structures wherein the crack is located closer to the stringer. (See the description of the test results in a subsequent section and Figure 9). 
The stress intensity factor history for the right crack tip is plotted in Figure 7 in terms of relative crack length $L / B$ where $L$ is the horizontal distance between crack tips and $B$ is the distance between stress intensity factor history can be identified:

- $K_{I}$ is the dominant stress intensity factor and increases as the crack grows.

- The ratio $K_{I I} / K_{I}$ becomes small after the crack is allowed to curve and remains small throughout crack growth. This behavior is known to occur for isotropic materials subjected to in-plane loading only [Wawrzynek (1991)].

- The ratio $k_{2} / K_{I}$ is small for the initial straight crack, but as the crack begins to curve, the value of $k_{2}$ increases such that the ratio of $k_{2} / K_{I}$ becomes large.

- The ratio $k_{1} / K_{I}$ remains small throughout crack growth.

The value of $k_{2}$ is affected by the resistance of opposing crack edges to out-of-plane deformation. Two trends are expected to occur: 1) as a longitudinal crack is moved closer to a stringer, the stiffness of the crack edge nearest to the stringer should increase relative to that of the opposing should diminish relative to that begins to curve, the stiffness of the crack edge on the concave side the longitudinal direction that restrict the edge on the convex side. (The tensile membrane stresses in concave side.). The initial crack in the trial prob cannot develop in the peninsula of material on the to be observed; however, it is observed in the validation problenough to the stringer for the first trend observed in both the deformed shapes of Figure 5 and the problem (see Figure 8). The second trend is as the crack curves, both the unsymmetric trial problem, the ratio of $k_{2} / K_{I}$ reaches 0.36 for the 14 inch

There is little symmetric bending occurring for the crack in the trial problem as indicated by the low value of $k_{1}$. The significant amount of membrane stretching occurring in the fuselage skin keeps this value low. The predominant crack tip loading consists of membrane stretching, characterized by $K_{I}$, and unsymmetric out-of-plane bending, characterized by $k_{2}$.

\section{Simulation 2: Validation Problem}

\section{Description of Experiment}

Two generic pressure test fixtures have been fabricated by the Boeing Commercial Airplane Group. The fixtures can be used for fatigue, fatigue crack growth, or residual strength tests. One fixture has a radius of curvature of 74 inches to match narrow-body airplanes, while the other has a radius of curvature of 127 inches to match wide-body airplanes. One end of each fixture is mounted in a rigid framework and the other on rollers to allow longitudinal displacement. Removable test sections are air as the pressurizing medium. More information. Tests are conducted under pressure loading only, using 
Experimental data generated using the test fixtures was made available to the authors by Boeing [Worden (1993)]. The data is from a typical narrow-body test panel with bonded tear straps. The panel had initial damage consisting of a 5.0 inch saw cut centered on the midbay tear strap and just above the stringer tear strap. The saw cut went completely through both the skin and the midbay tear strap. The panel was inserted into the test fixture and pressure cycled at $7.8 \mathrm{psi}$. The pressure cycles fluctuated between maximum pressure and zero pressure. During the test, the position of each crack tip was monitored and recorded along with the number of pressure cycles. The experimental data can be found in Potyondy (1993) and Potyondy, et al. (1994b).

\section{Description of Simulation}

The test fixture approximates the behavior of an actual pressurized fuselage. Thus, a cracked pressurized fuselage is modeled using the analysis methodology described above. The validation problem differs from the trial problem only in the following details: 1 ) initial crack size and location; and, 2) boundary conditions applied to the global shell model. The initial straight 5.0 inch crack is located in a panel that is representative of the fuselage crown. In both the global shell model and the $6 \times 6$ bay stiffened panel model, the initial crack is located in the same location as the saw cut. In the FRANC3D model, the initial crack has been translated a distance of 0.45 inches (half the stringer width) away from the stringer, because the current version of FRANC3D cannot model cracks at the interface of the stringer and skin.

The boundary conditions applied to the global shell model are a uniform internal pressure of 7.8 psi and no mechanical loading. A plane of symmetry is assumed at one end of the barrel while the other end is constrained to remain in a plane. Note that this model corresponds with an open cylinder, and thus the longitudinal stress in the model is less than the longitudinal stress in the test fixture, since the test fixture is a closed cylinder. The crack is grown in one inch increments at each crack tip up to a total piecewise crack length of 19 inches.

The deformed structure for various crack lengths is shown in Figure 8. The computed crack trajectory is plotted in Figure 9 along with the measured trajectory from the panel test. The computed stress intensity factor history for the right crack tip is plotted in Figure 10. The stress intensity factor history at the left crack tip is similar.

There is no analytical solution with which the computed stress intensity factors can be compared. However, the computed values of $K_{I}$ can be compared to those obtained from the finite element analysis of Miller, et al. (1992) of a similar panel test. The comparison is described in Potyondy (1993) and Potyondy, et al. (1994b) where it is shown that the trends and values from both analyses are similar.

Fatigue Life Prediction. A life prediction has been made using the computed stress intensity factor history and a Paris fatigue crack growth model. Fatigue crack growth data from a flat 2024-T3 panel tested at stress intensity factors in the range of 50-80 ksi-sqrt (in) was made available to the authors by Boeing [Worden (1993)]. The crack growth equation is of the form

$$
(10,000) \frac{d a}{d N}=\left(\frac{K_{\max }}{C}\right)^{n}
$$


and for 2024-T3 aluminum T-L crack growth ( $R=0$ and ambient lab environment)

$$
\begin{aligned}
& C=28 \\
& n=4.9
\end{aligned} ; \text { for } K_{\max }(\mathrm{ksi} \sqrt{\mathrm{inch}})
$$

By using the computed values of $K_{I}$, and assuming that $K_{I}$ varies linearly between the data points in Figure 10, life predictions were made and are compared with measured life in Figure 11 ( $K_{\text {eff }}$ is defined below by Eq. (6); for $\alpha=0, K_{e f f}=K_{I}$ ).

The total predicted life is obtained by integrating the crack growth model until $K_{I}$ reaches $K_{a p p}$, the apparent toughness of the material. $K_{a p p}$ is a function of thickness and crack orientation relative to material rolling direction. The values of $K_{a p p}$ for this material and thickness are

$$
\begin{array}{ll}
K_{a p p}=100 \mathrm{ksi} \sqrt{\text { inch }} \quad & \text { (T - L crack growth) } \\
K_{a p p} \approx 105-120 \mathrm{ksi} \sqrt{\text { inch }} & (\mathrm{L}-\mathrm{T} \text { crack growth) }
\end{array}
$$

T-L crack growth refers to the crack aligned with the rolling direction, while L-T crack growth refers to the crack perpendicular to the rolling direction. For the panel tested, the rolling direction is the longitudinal direction; thus, cracks growing from the initial saw cut are experiencing T-L crack growth. The computed value of $K_{I}$ for $L / B=0.77$ is $112 \mathrm{ksi}$-sqrt (in). At this point, the crack has curved considerably and is oriented at nearly 45 degrees with respect to the two orthogonal rolling directions; thus, the value of $K_{a p p}$ will lie within the range of T-L and L-T crack growth. For the purposes of making a total life prediction, it is assumed that $K_{I}=K_{a p p}$ for $L / B=0.77$.

Discussion of Fatigue Life Prediction. The life prediction for the validation problem compares well with the measured life. For all crack lengths considered in the simulation, the predicted life is never more than 25 percent greater than the measured life. In addition, the total life prediction is 628 cycles. In the panel test, crack growth ceased after 626 cycles because of the large flap that had formed in the skin making further pressurization impossible. Thus, the total predicted life is in excellent agreement with the measured life; however, such an extremely close comparison is surely serendipitous.

Life predictions within a factor of two are considered good for this test for the following reasons:

- The crack growth rates throughout the test are high; thus, the predicted life is sensitive to small changes in the computed values of $K_{I}$ because of the large coefficient of 4.9 in the Paris crack growth model.

- During the final stages of crack growth, the computed values of $K_{I}$ are approaching $K_{\text {app }}$; thus, the crack growth is about to become unstable. It is likely that large plastic zones were present at the crack tips, thereby casting doubt on applicability of the LEFM assumptions. However, this effect is offset by the use of Paris model coefficients that were obtained for stress intensity factors above $50 \mathrm{ksi}$-sqrt (in).

A final observation is that the prediction of slower crack growth than observed supports the hypothesis that the presence of $k_{2}$ will increase the crack growth rates from those obtained by 
considering only $K_{I}$. It is expected that the experimental work of Zehnder, et al. (1992) and Viz and Zehnder (1994) will shed more light on this issue and may result in a modified procedure for determining life. In lieu of such a procedure, a simple empirical procedure for incorporating and bounding the effect of the additional stress intensity factors on the crack growth rate is proposed here. It assumes that a fraction of the energy from the additional stress intensity factors contributes to fatigue crack growth. Replace the value of $K_{I}$ in the fatigue crack growth model with an effective stress intensity factor $K_{e f f}$, that is related to an effective energy release rate $G_{e f f}$, via

$$
\begin{gathered}
K_{\text {eff }}=\sqrt{E G_{\text {eff }}} \\
G_{\text {eff }}=G_{1}+\alpha\left(G_{2}+G_{3}+G_{4}+G_{5}\right) \quad ; \quad 0 \leq \alpha \leq 1.0
\end{gathered}
$$

where $E$ is Young's modulus, $G_{i}$ are the separate components of the energy release rate that are related to the four stress intensity factors by Eq. (1), and $\alpha$ is an empirical coefficient that scales the contribution from the additional stress intensity factors.

The empirical procedure is applied to the validation problem, and the resulting life predictions for $\alpha$ equal to 0.0 and 1.0 (expected lower and upper bounds) are compared with measured life in Figure 11. The $\alpha$ coefficient shifts each computed data point to the left by a fixed amount, e.g., the last computed data point will coincide with the measured value by setting $\alpha=0.4$. Also, total life is decreased as $\alpha$ is increased. During crack growth, although the ratio of $k_{2} / K_{I}$ increases by almost 40 percent, the contribution of $K_{I}$ to the total energy release rate decreases by less than 4 percent suggesting that $k_{2}$ may have a minor effect on crack growth for this class of problems.

\section{Discussion}

The present analysis methodology allows the cracks to curve and accounts for the nonlinear bulge-out effect and the presence of stiffening elements by using a geometrically nonlinear solution procedure. Also, the interaction between the mechanical loads acting on the superstructure and the local structural response near the crack tips is accounted for by employing a hierarchical modeling strategy. The computed stress intensity factors exhibit the expected trends and their values are reasonable. The computed crack trajectory and predicted life agree well with the measured quantities from a full-scale pressurized panel test. The effect of the additional stress intensity factors, in addition to $K_{I}$, on crack growth rate can be incorporated into a life prediction using an effective stress intensity factor.

The unsymmetric, out-of-plane deformation experienced by a crack in a pressurized fuselage structure can be seen clearly in the geometrically nonlinear finite element models (see Figures 5 and 8 ) and can be accounted for by the computation of $k_{2}$. However, the effect of $k_{2}$ on crack growth rate is still uncertain. It is expected that further testing by Zehnder, et al. (1992) and Viz and Zehnder (1994) will determine this effect. The slight underprediction of crack growth rate for the validation problem may indicate that the presence of $k_{2}$ increases the crack growth rate beyond that obtained by only considering $K_{I}$; however, this result is preliminary. Other factors, such as the lack of applying a longitudinal loading to the global shell model, may also account for the slight underprediction of crack growth rates. Further comparisons between predicted and measured lives must be made before more definitive conclusions can be drawn. 
The maximum tangential stress crack growth directional criterion used here predicts a crack trajectory in good agreement with the measured trajectory from a full-scale pressurized panel test (see Figure 9). Depending upon the accuracy required in the trajectory determination, this simple criterion may be sufficient for most practical situations.

The fact that the boundary conditions on the $2 \times 2$ bay stiffened panel model were not altered during crack growth; i.e., the crack was only grown in the $2 \times 2$ bay model, and did not seriously affect the accuracy of the trajectory and life predictions. The kinematic boundary conditions are affected minimally by crack growth near the center of the panel and must only be updated when the crack begins to approach the panel boundary. The crack behavior is affected to a much greater extent by the live pressure loading, and the effect of this loading is being recomputed after each step of crack growth.

Restarting the geometrically nonlinear finite element computation from zero load after each crack growth increment requires a significant computational effort. The computational efficiency could be improved by using the previously computed equilibrium state as an initial condition for the succeeding computation after the crack has grown. The procedure is described in the paper of Potyondy and Ingraffea (1992). Its implementation would reduce the total computation time to perform a crack
growth simulation significantly.

It is known that fastener flexibility alters the stress distribution locally in the fuselage skin which influences the behavior of cracks in these regions. The effect of neglecting fastener flexibility in the current analysis methodology should be examined. Also, the repeatability of the panel test experiments should be determined along with the effect of small variabilities, such as fit-up stresses and rivet forces, on crack growth behavior.

\section{CONCLUSIONS}

The present methodology provides an effective engineering approach to simulate crack growth in pressurized aircraft fuselages where crack trajectory is not know a priori. It accounts for the dominant influence factors affecting crack growth behavior (the bulge-out effect; presence of stiffening elements; and, mechanical loads, in addition to the internal pressure, acting on the superstructure) and integrates them with a set of LEFM fracture parameters to support trajectory and life predictions. The predicted crack trajectory and life agree well with the measured quantities from a full-scale pressurized panel test indicating the effectiveness of the methodology. A logical extension of the present methodology would be to account for plasticity effects at the crack tips, as these effects may become significant for long cracks encountered during residual strength computations.

\section{ACKNOWLEDGEMENTS}

This work was performed with support from the NASA Langley Aircraft Structural Integrity Program under contract NAG-1-1184. Ms. Vicki Britt of the NASA Langley Aircraft Structures Branch performed the global shell analyses to provide the boundary conditions for the FRANC3D models of the 
example simulations. Dr. Charles Rankin, Dr. Frank Brogan, and Mr. Harold Cabiness of the Lockheed Palo Alto Research Laboratory provided the STAGS shell finite element code. Dr. Matthew Miller and Dr. R. Elaine Worden of the Boeing Commercial Airplane Group provided the structural details and dimensions of the typical narrow-body fuselage panel and the experimental data of the full-scale pressurized panel test. And finally, the authors would like to thank Prof. Alan Zehnder, Mr. Mark Viz, and Dr. Bruce Carter for many helpful discussions.

\section{REFERENCES}

Almroth, B.O., Brogan, F.A., and Stanley, G.M., 1986, "Structural Analysis of General Shells," User's Instructions for STAGSC-1, Vol. 2, Lockheed Missiles and Space Company, LMSC D633873.

Erdogan, F., and Sih, G.C., 1963, “On the Crack Extension of Plates under Plane Loading and Transverse Shear," Journal of Basic Engineering, 85, No. 4, pp. 519-527.

Gray, L.J., Potyondy, D.O., Lutz, E.D., Wawrzynek, P.A., Martha, L.F., and Ingraffea, A.R., 1994, "Crack Propagation Modeling," to appear in Mathematical Models and Methods in Applied Sciences.

Hui, C.Y., and Zehnder, A.T., 1993, "A Theory for the Fracture of Thin Plates Subjected to Bending and Twisting Moments," International Journal of Fracture, Vol. 61, pp. 211-229.

Maclin, J.R., 1991, “Performance of Fuselage Pressure Structure,” Proc. of 1991 International Conference on Aging Aircraft and Structural Airworthiness, Washington, D.C. (November 19-21, 1991), C.E. Harris, ed., NASA Conference Publication 3160, pp. 67-74.

Martha, L.F., 1989, "Topological and Geometrical Modelling Approach to Numerical Discretization and Arbitrary Fracture Simulation in Three-Dimensions," Ph.D. Thesis, Cornell University, Ithaca, NY.

Martha, L.F., Wawrzynek, P.A., and Ingraffea, A.R., 1993, "Arbitrary Crack Representation Using Solid Modeling," Engineering with Computers, Vol. 9, pp. 63-82.

Miller, M., Kaelber, K.N., and Worden, R.E., 1992, "Finite Element Analysis of Pressure Vessel Panels," Durability of Metal Aircraft Structures : Proc. of International Workshop on Structural Integrity of Aging Airplanes, S.N. Atluri, C.E. Harris, A. Hoggard, N. Miller, and S.G. Sampath, eds., Atlanta Technology Publications, Atlanta, GA, pp. 337-348.

Potyondy, D.O., and Ingraffea, A.R., 1992, “A Methodology for Simulation of Curvilinear Crack Growth in Pressurized Fuselages," Durability of Metal Aircraft Structures : Proc. of International Workshop on Structural Integrity of Aging Airplanes, S.N. Atluri, C.E. Harris, A. Hoggard, N. Miller, and S.G. Sampath, eds., Atlanta Technology Publications, Atlanta, GA, pp. $217-230$.

Potyondy, D.O., Gray, L.J., and Ingraffea, A.R., 1992, "Simulation of 3D Non-planar Fatigue Crack Growth in a Turbine Blade Root," Computer Technology: Advances and Applications : Proc. of ASME Pressure Vessels and Piping Conference, R.S. Gallagher and G.M. Hulbert, eds., PVP-Vol. 234, ASME, New York, pp. 31-42.

Potyondy, D.O., 1993, “A Software Framework for Simulating Curvilinear Crack Growth in Pressurized Thin Shells," Ph.D. Thesis, Cornell University, Ithaca, NY. 
Potyondy, D.O., Rankin, C.C., Riks, E., Viz, M.J., and Zehnder, A.T., 1993, "Computation of Applied

Potyondy, D.O., Wawrzynek, P.A., and Ingraffea, A.R., 1994a, “An Algorithm to Generate Quadrilateral or Triangular Element Surface Meshes in Arbitrary Domains With Applications to Crack Propagation," submitted to International Journal of Numerical Methods in Engineering.

Potyondy, D.O., Wawrzynek, P.A., and Ingraffea, A.R., 1994b, "Discrete Crack Growth Analysis Methodology for Through Cracks in Pressurized Fuselage Structures," submitted to International Journal of Numerical Methods in Engineering.

Rankin, C.C., and Brogan, F.A., 1991, "The Computational Structural Mechanics Testbed Structural Element Processor ES5: STAGS Shell Element," NASA Contractor Report 4358, Lockheed Missiles and Space Company, LMSC-D878511.

Rankin, C.C., Brogan, F.A., and Riks, E., 1993, "Some Computational Tools for the Analysis of Through Cracks in Stiffened Fuselage Shells," Journal of Computational Mechanics, Vol. 13, pp.

Shenoy, V.B., Potyondy, D.O., and Atluri, S.N., 1994, “A Methodology for Computing Nonlinear Fracture Parameters for a Bulging Crack in a Pressurised Aircraft Fuselage," to appear in Journal of

Starnes, J.H., and Britt, V.O., 1991, "Damaged Stiffened Shell Research at NASA Langley Research Center," Proc. of 1991 International Conference on Aging Aircraft and Structural Airworthiness,

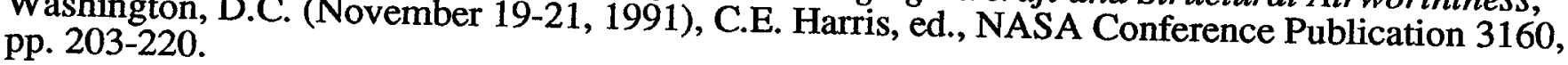

Viz, M.J. and Zehnder, A.T., 1994, "Fatigue Crack Growth in 2024-T3 Aluminum Under Tensile and Tearing Loads," FAA/NASA Intnl. Symposium: Advanced Structural Integrity Methods for Airframe Durability and Damage Tolerance, NASA CP-3274, 1994.

Wawrzynek, P.A., Martha, L.F., and Ingraffea, A.R., 1988, "A Computational Environment for the Simulation of Fracture Processes in Three Dimensions," Analytical, Numerical, and Experimental Aspects of Three Dimensional Fracture Processes, A.J. Rosakis, et al., eds., ASME, New York,

Wawrzynek, P.A., 1991, "Discrete Modelling of Crack Propagation: Theoretical Aspects and Implementation Issues in Two and Three Dimensions, "Ph.D. Thesis, Cornell University, Ithaca

Worden, R.E., 1993, private communication from Boeing Commercial Airplane Group.

Zaal, K.J.J.M., 1992, “A Survey of Crack Path Stability Theories with an Eye to an Application to Crack Flapping Phenomena in Pressurized Fuselages," Faculty of Aerospace Engineering, Delft University, Delft, The Netherlands, Report LR-681.

Zehnder, A.T., Viz, M.J., and Ingraffea, A.R., 1992, "Fatigue Fracture in Thin Plates Subjected to Tensile and Shearing Loads: Crack Tip Fields, J Integral and Preliminary Experimental Results," Mechanics, Las Vegas, Nor Experimental Mechanics International Congress on Experimental 


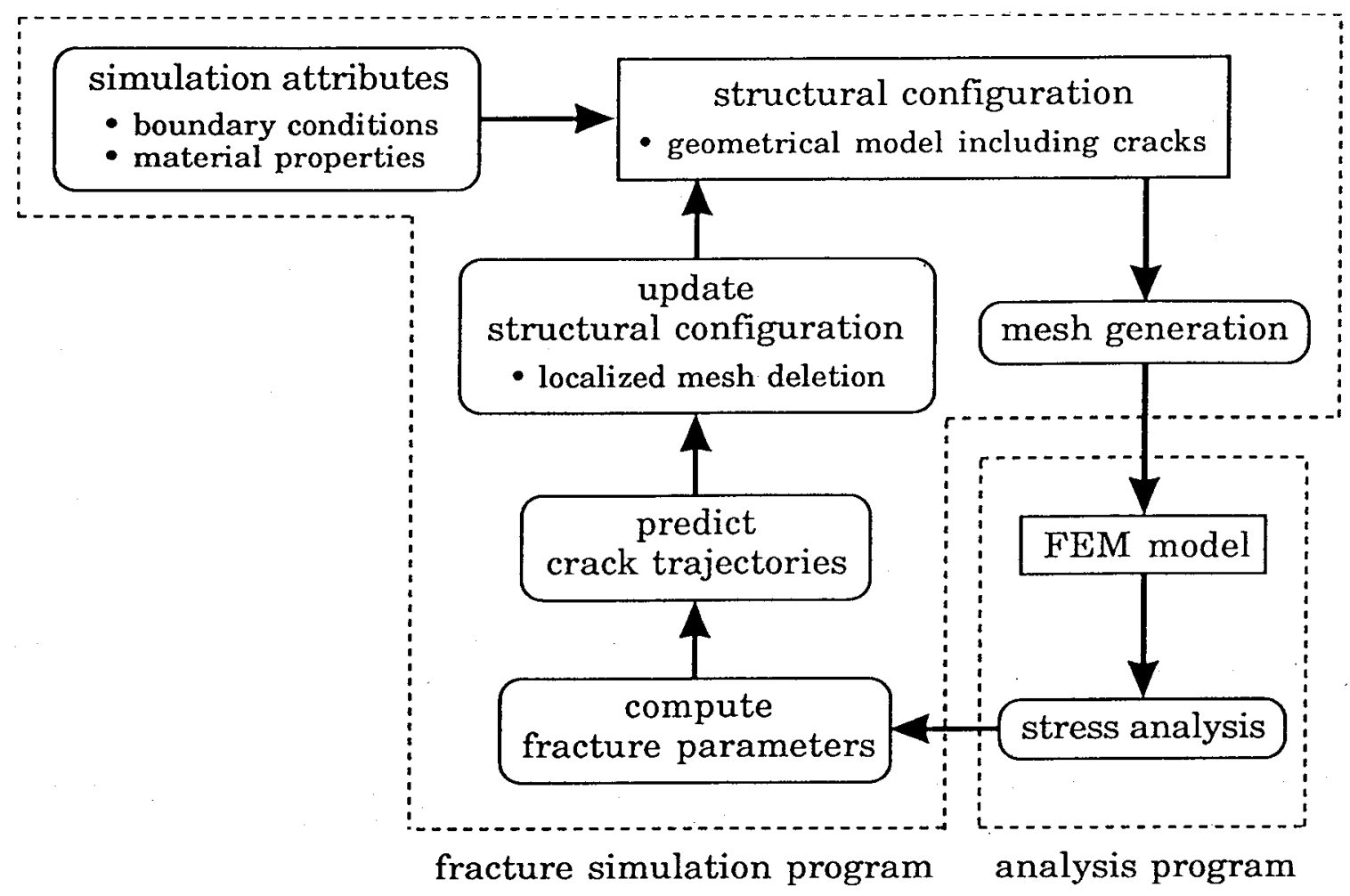

Figure 1: Discrete crack growth analysis methodology.

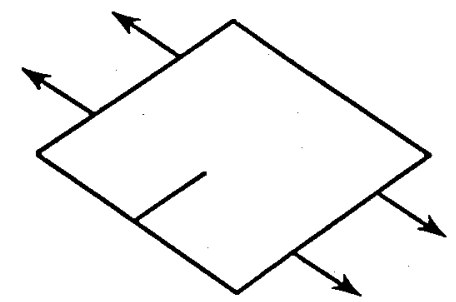

Symmetric membrane loading, $K_{I}$

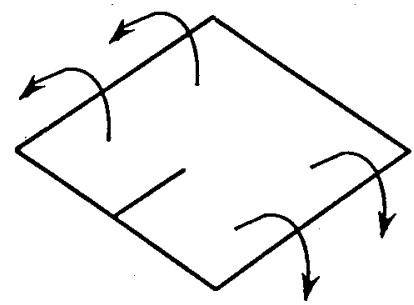

Symmetric bending loading, $k_{1}$

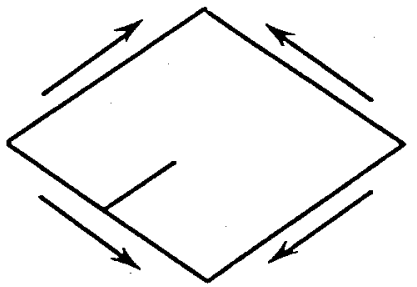

Anti-symmetric membrane loading, $K_{I I}$

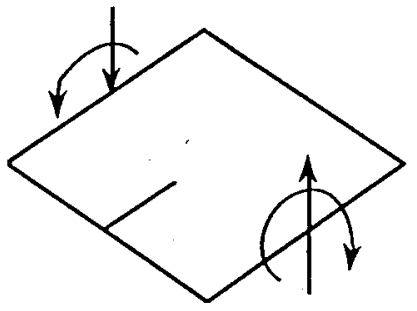

Anti-symmetric bending loading, $k_{2}$

Figure 2: The four loading modes and their corresponding stress intensity factors for a through crack in a thin plate idealized using plane stress elasticity to account for the membrane loads and Kirchhoff plate theory to account for the bending loads. 


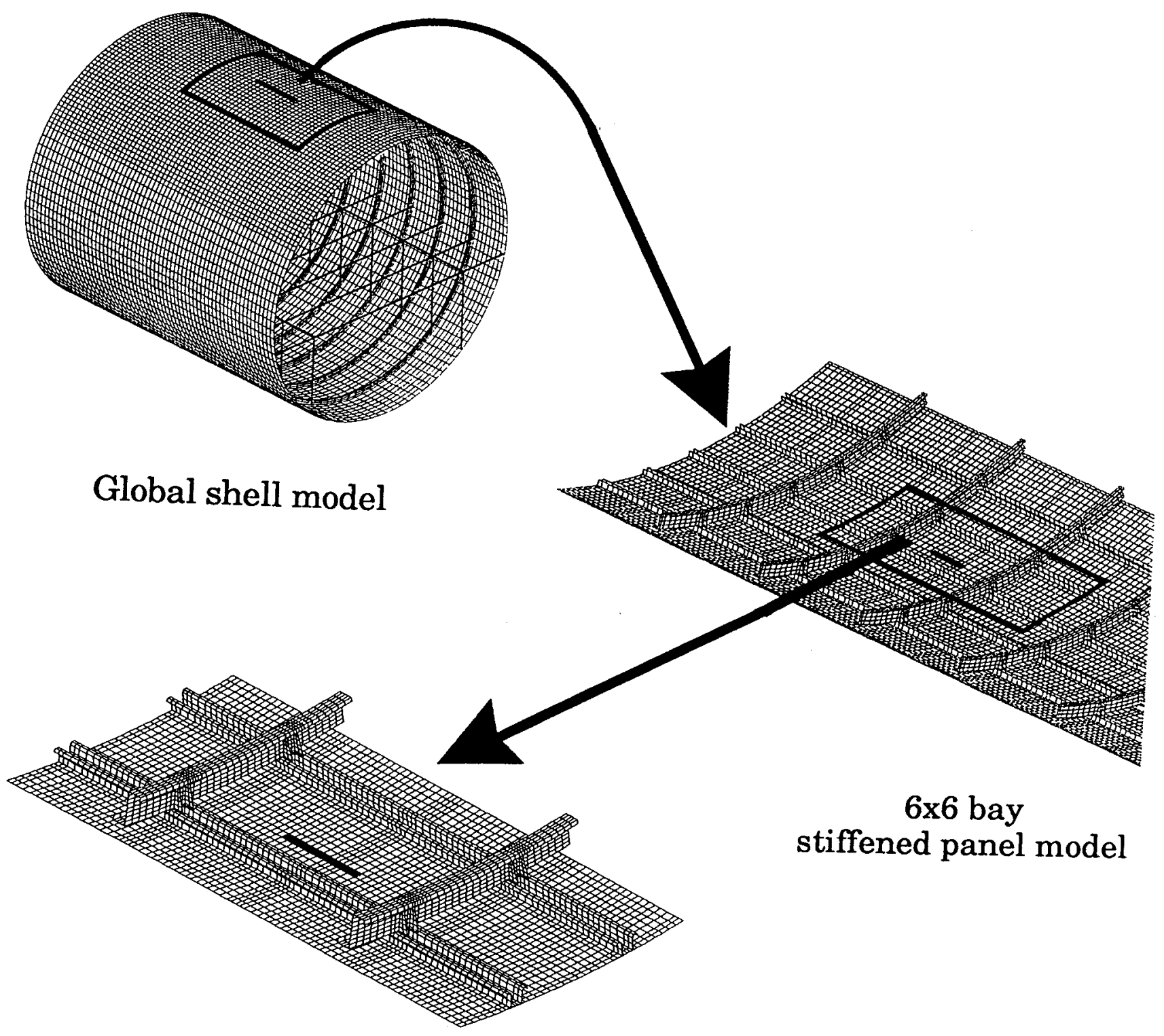

$2 \times 2$ bay stiffened panel model

Figure 3: The three hierarchical modeling levels. Each model obtains its kinematic boundary conditions from the preceeding model in the
hierarchy. 


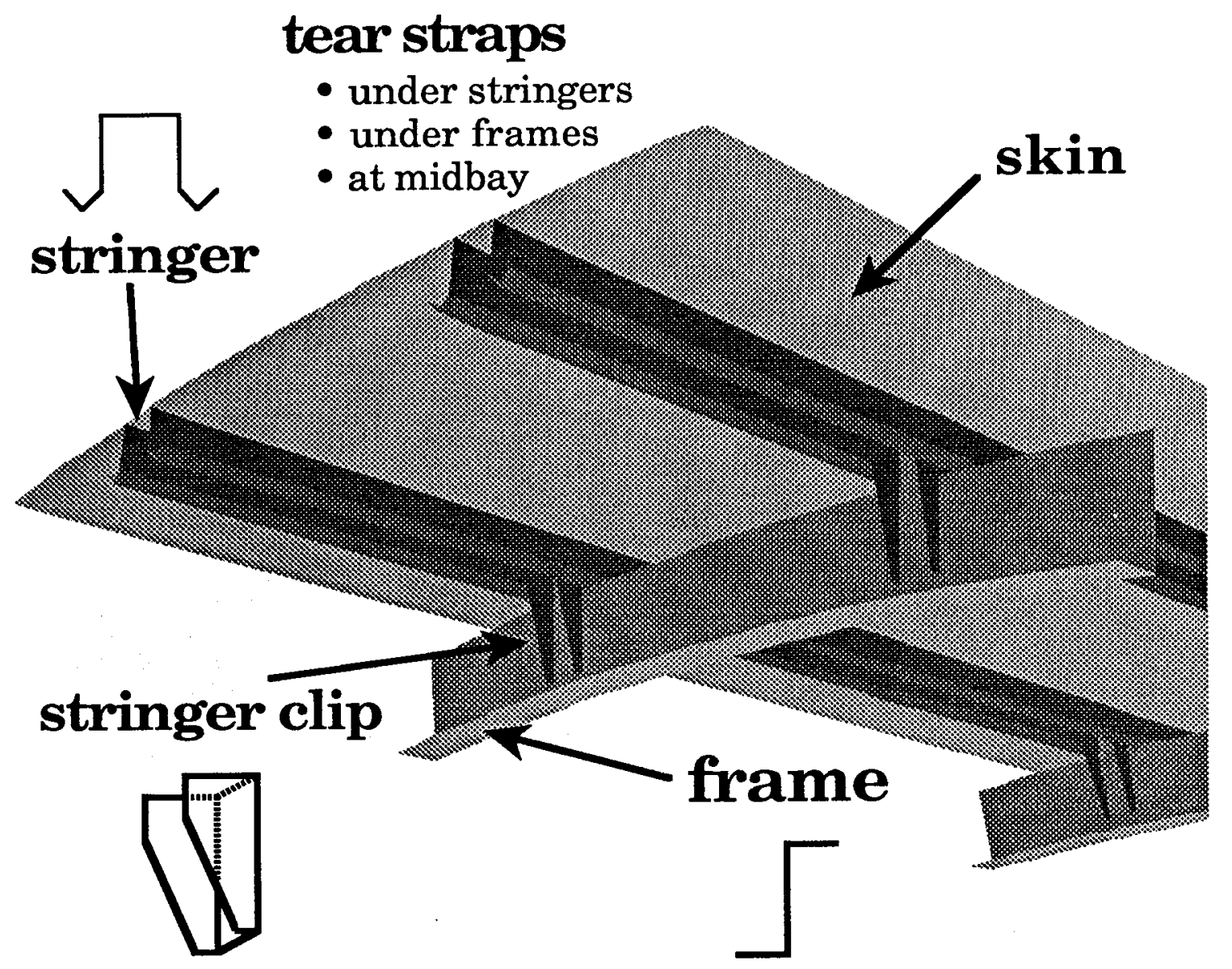

Figure 4: The $2 \times 2$ bay stiffened panel model as represented within FRANC3D as a geometrical model. The structural components and their cross sections are labelled. 

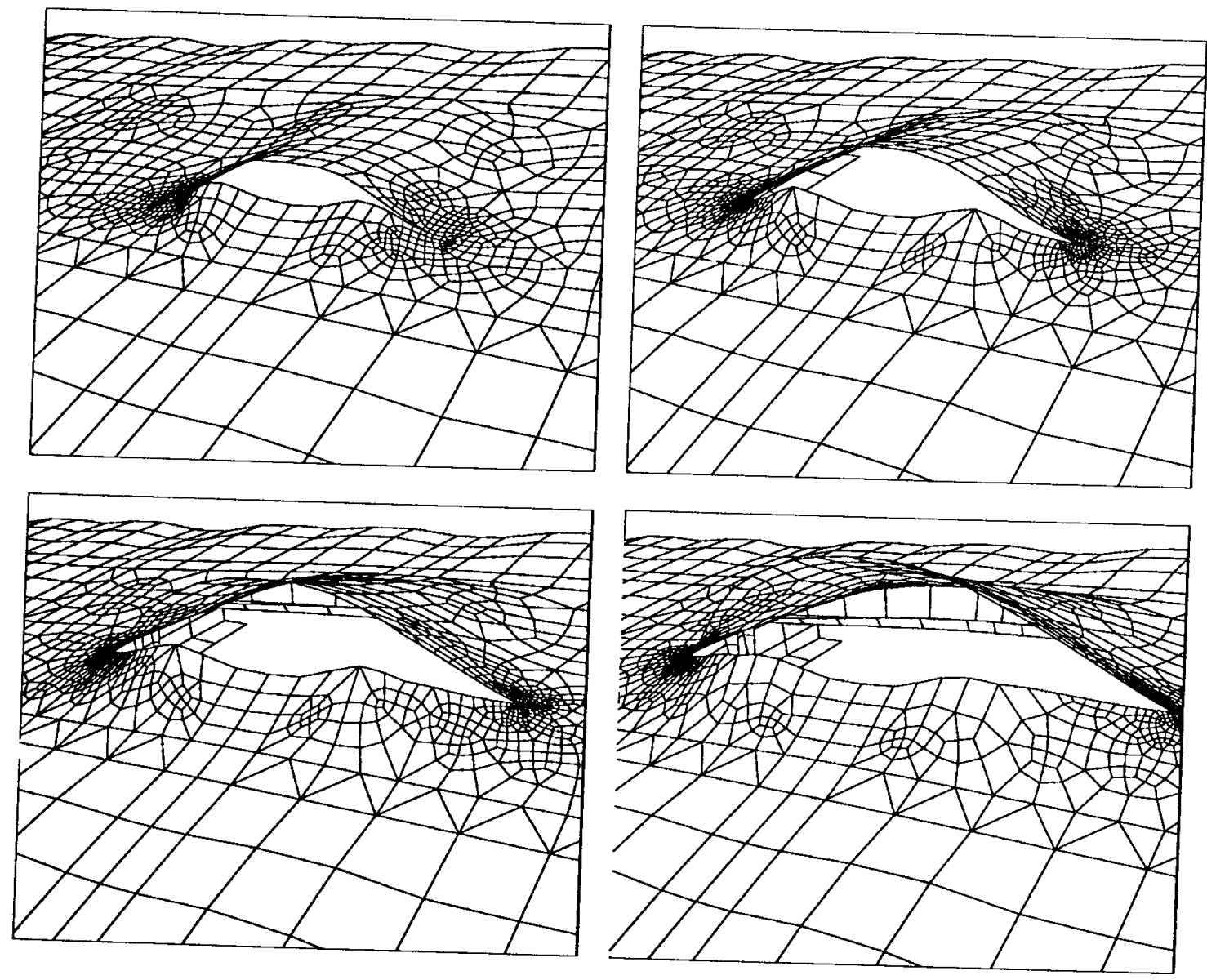

Figure 5: Deformed structure during crack growth for total crack lengths of 6,8, 10 , and 12 inches for the trial problem. Magnification factor for all
images is five. 


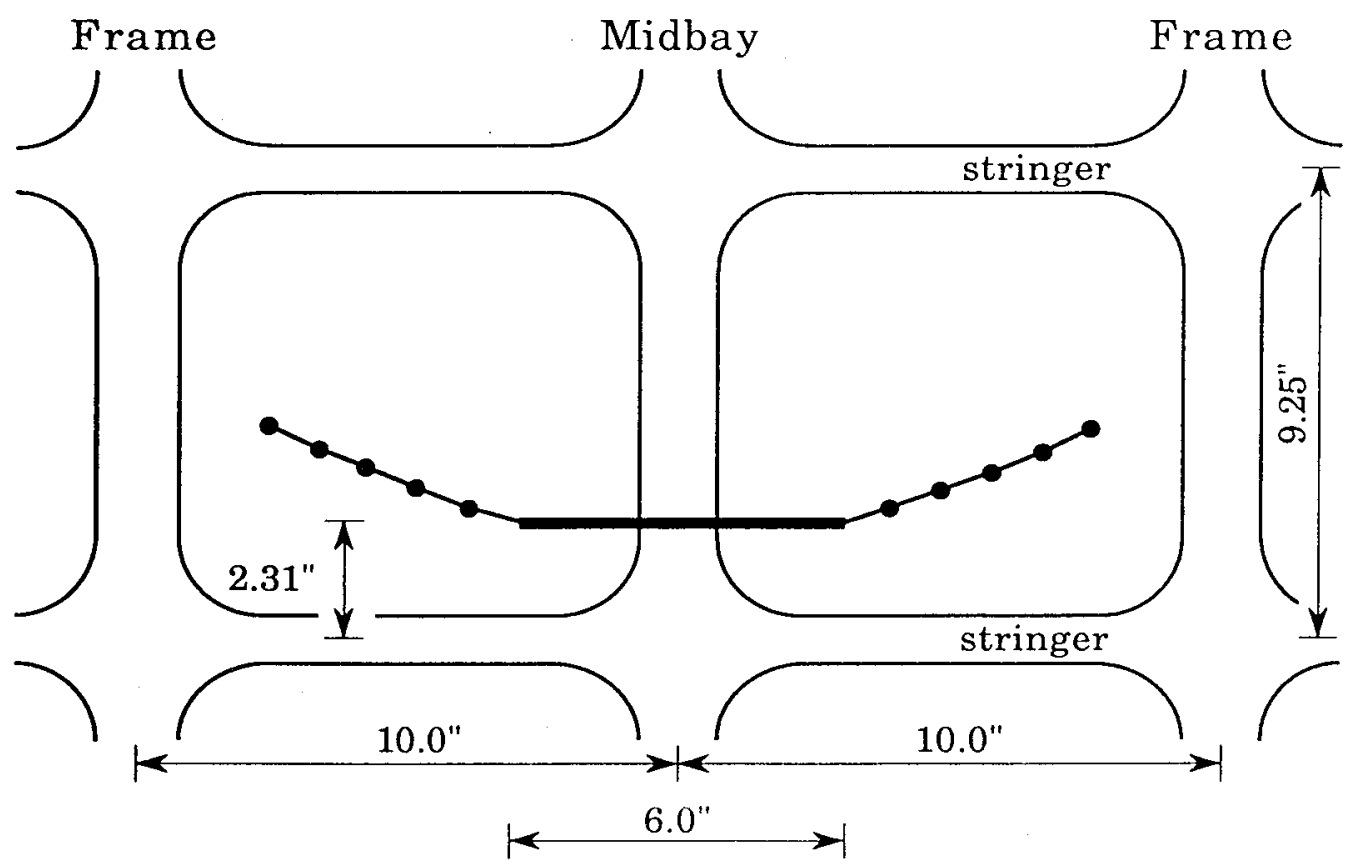

Figure 6: Computed crack growth trajectory for the trial problem. The black circles denote computed crack tip locations.

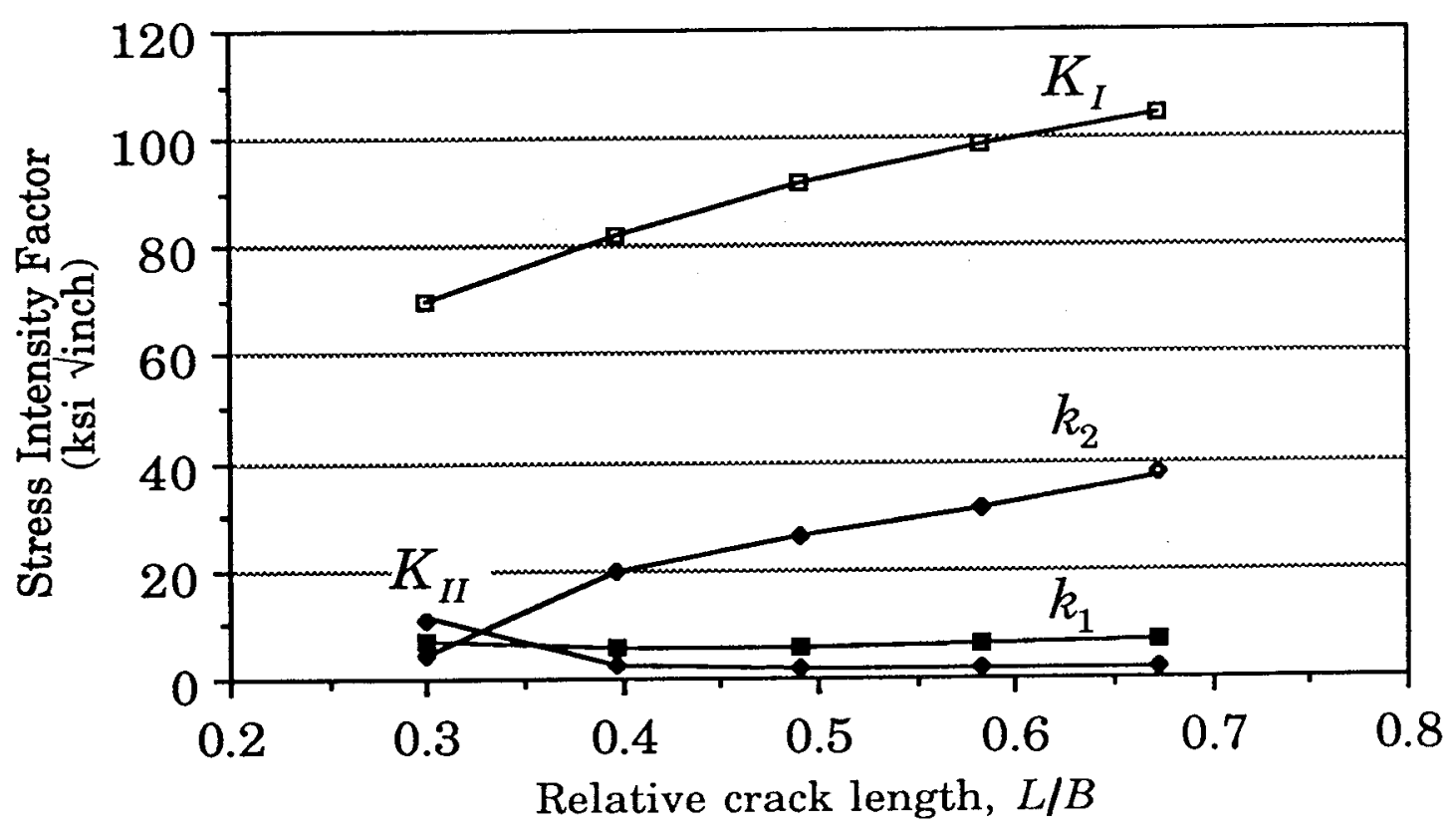

Figure 7: Computed stress intensity factors at right crack tip for the trial problem. 

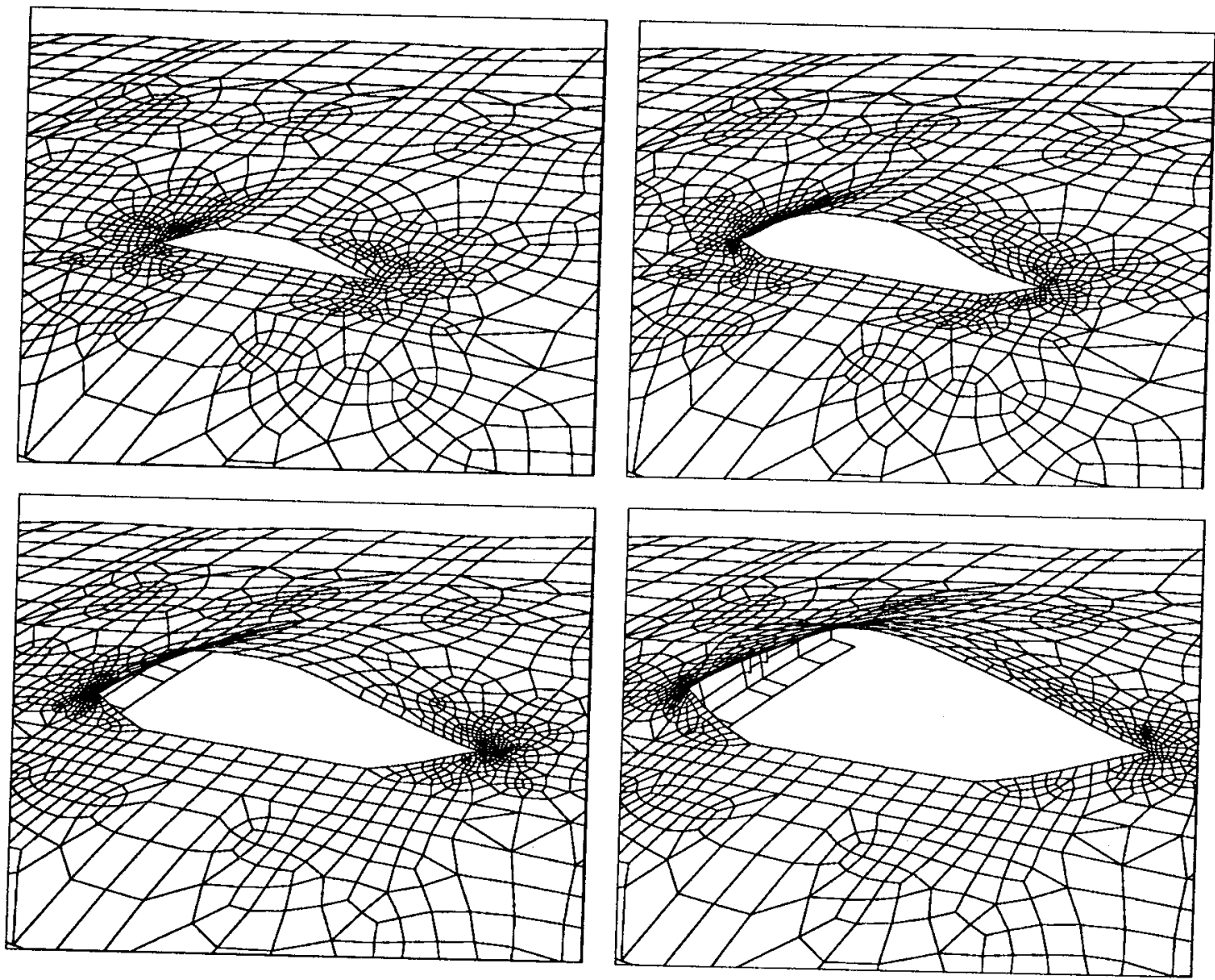

Figure 8: Deformed structure during crack growth for total crack lengths of 5, 7, 9, and 11 inches for the validation problem. Magnification factor for all
images is five. 


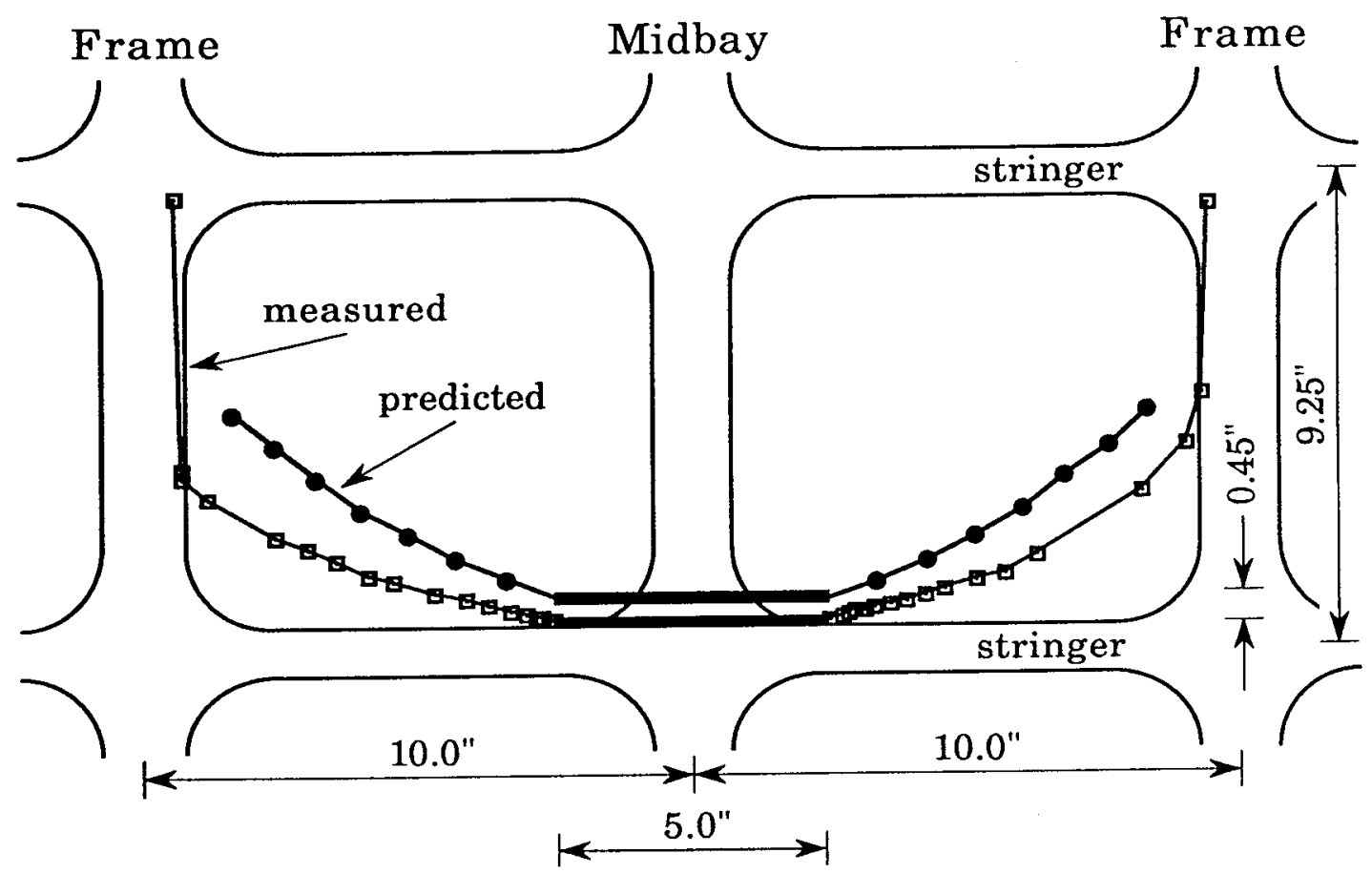

Figure 9: Comparison between computed and measured crack growth trajectories for the validation problem. The black circles and open boxes denote computed and measured crack tip locations, respectively.

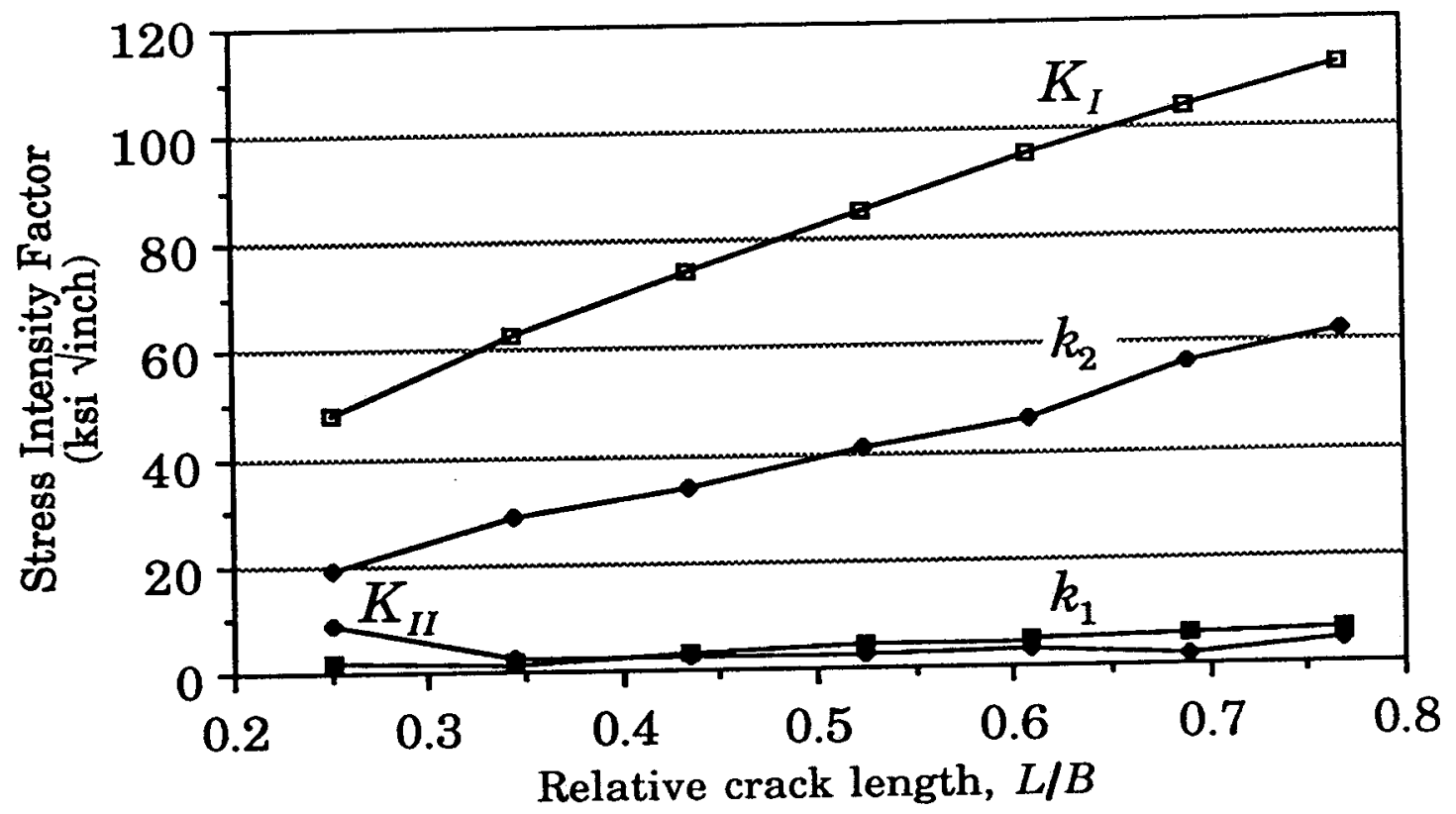

Figure 10: Computed stress intensity factors at right crack tip for the validation problem. 


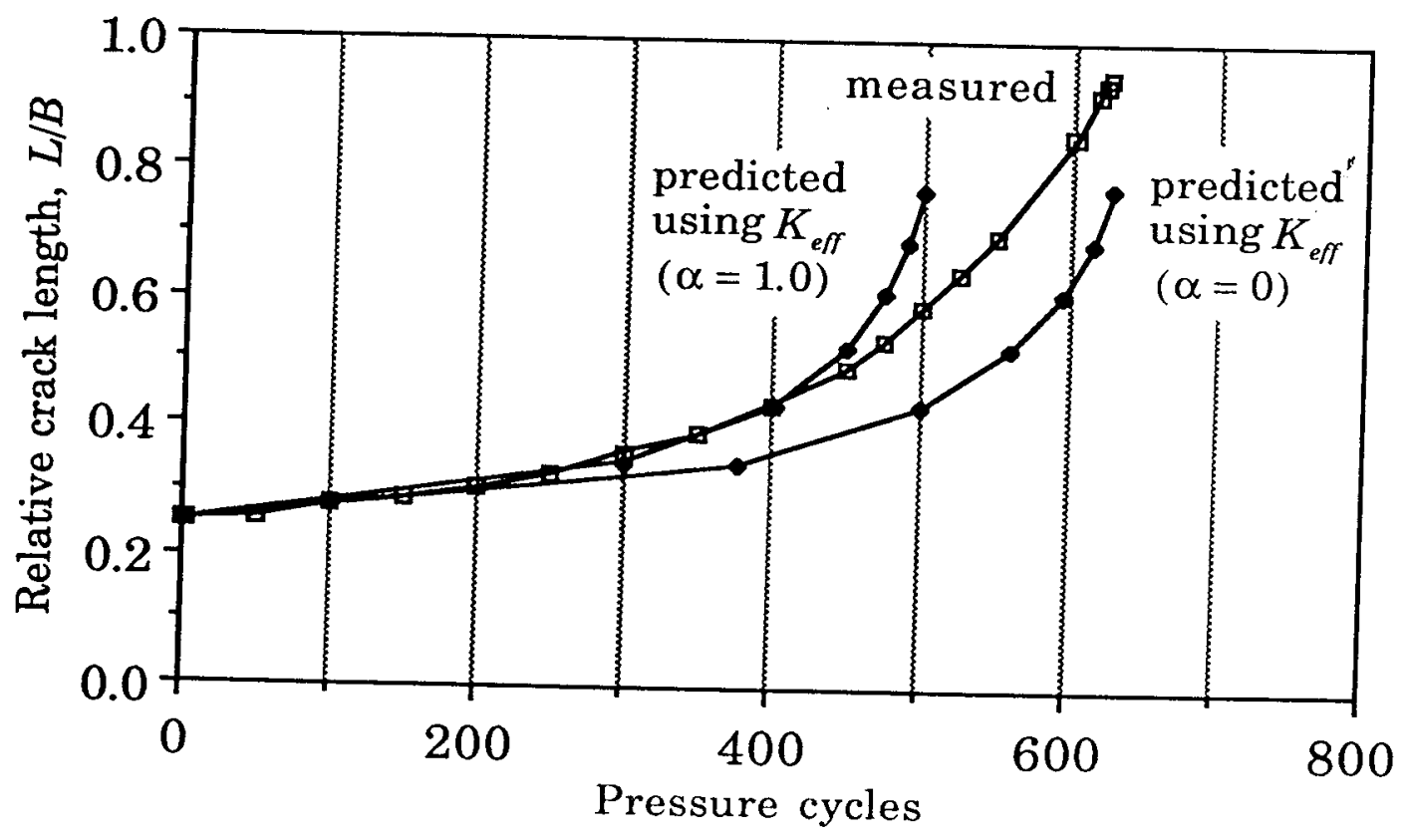

Figure 11: Comparison between predicted and measured fatigue life. The life prediction is made using the effective stress intensity factor of Eq. (6). 